\title{
Laser scattering characteristics of common roads (Withdrawal Notice)
}

\section{Yuxiang Jiang, Zhenhua Li}

Yuxiang Jiang, Zhenhua $\mathrm{Li}$, "Laser scattering characteristics of common roads (Withdrawal Notice)," Proc. SPIE 11552, Optical Metrology and Inspection for Industrial Applications VII, 115521M (10 October 2020); doi:

10.1117/12.2575112

SPIE. Event: SPIE/COS Photonics Asia, 2020, Online Only 


\section{Laser scattering characteristics of common roads (Withdrawal Notice)}

Yuxiang Jiang, Zhenhua Li

Nanjing Univ. of Science and Technology (China)

Proceedings Volume 11552, Optical Metrology and Inspection for Industrial Applications VIl; $115521 \mathrm{M}$ (2020) https://doi.org/10.1117/12.2575112 Event: SPIE/COS Photonics Asia, 2020, Online Only

Event: SPIE/COS Photonics Asia, 2020, Online Only

Online Publication Date: 10 October 2020

Withdrawn from Publication: 2 February 2021

Publisher's Note: This paper, originally published on 10 October 2020, was withdrawn per author request. 